\title{
Une année déjà !
}

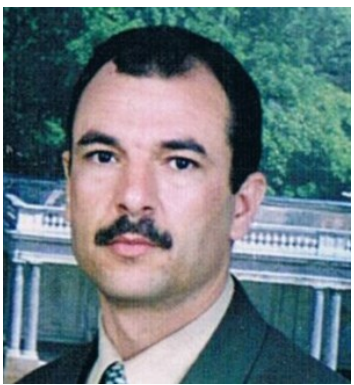

Correspondance à :

Dr. Abdelhak ABABSA MOUAKI hhakou2002@yahoo.fr

DOI:https://doi.org/10.48087/ BJMSe.2014.1201

\section{Chers lecteurs, chères lectrices,}

Comme suite logique nous vous livrons le deuxième numéro de Batna journal of medical sciences, et aussi avec ce numéro 2 de l'année 2014, la revue souffle sa première bougie et entame un nouveau tournant grâce à de nouvelles perspectives. Ce passage de témoin a également été l'occasion d'un renforcement du comité éditorial composé essentiellement de chercheurs et d'enseignants-chercheurs.

Tout le comité éditorial se joint à moi pour rendre hommage au travail considérable des docteurs Samy Slimani, rédacteur en chef et Hocine Gacem, rédacteur en chef adjoint, ainsi que d'autres collaborateurs, qui au cours de cette année 2014 avaient notamment maintenu le régime de parution des numéros de notre revue, professionnalisé sa mise en forme, animé le comité éditorial et assuré de nombreuses tâches ingrates relatives à la parution d'une revue médicale dans un contexte difficile. Sans eux, la revue n'aurait pu se maintenir.

Cette renaissance de la revue médicale de Batna n'aurait pas non plus été possible sans le soutien des Drs Mourad Djebbar, Imen Bencharif, Assia Haddouche, Adlen Nezzar, Amina Aggabi ... et d'autres qui avaient déjà donné une nouvelle impulsion à la revue dans sa première année d'existence.

Ce numéro régulier, le deuxième, de Batna journal of medical sciences et le troisième si l'on compte le supplément paru en septembre, témoigne de la vitalité d'une publication qui a su trouver sa place dans l'espace algérien de la référence tout en illustrant sa diversité et ses indispensables échanges avec la recherche. Et comme dans chaque numéro régulier, un dossier médical vient éclairer un questionnement plus général ; celui de ce numéro est consacré aux interactions médicamenteuses, dossier parrainé par le Dr Hocine Gacem et ayant fait intervenir des experts en pharmacologie des quatre coins du pays (Alger, Annaba, Batna, Oran, Sidi Bel-Abbès, Tlemcen).
Aussi, une des nouveautés de ce numéro réside dans l'intégration d'un nouveau type d'articles, les « arrêts sur image » qui illustrent dans un message concis et simple, un aspect inhabituel observé dans notre pratique médicale algérienne. Nous vous invitons à assurer sa pérennité en l'enrichissant par votre propre expérience.

Je ne peux pas m'empêcher de vous informer de la création récente d'une page Facebook dédiée à la revue, retrouvée sur le lien : www.facebook.com/batnajms. En deux semaines, plus de 600 personnes ont «aimé » la page, ce qui permettra d'augmenter sa visibilité et faciliter l'accès aux articles, déjà aisé par la disponibilité gratuite de tous les articles sur le site web de la revue : http://batnajms.com.

Bonne lecture et rendez-vous en 2015 pour le prochain numéro.

Dr Abdelhak ABABSA MOUAKI Rédacteur en Chef Adjoint

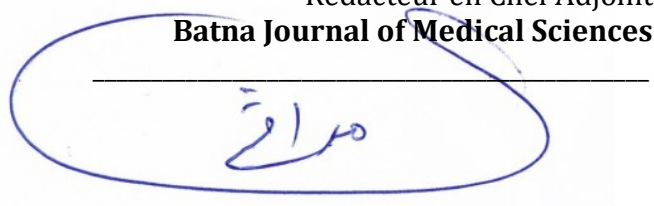

Ababsa Mouaki A. Une année déjà ! Batna J Med Sci 2014;1(2):47. https://doi.org/10.48087/ B]MSe.2014.1201 